\title{
Contents, Vol. 13, 1987
}

\section{Editor-in-Chief Editorial Board}

E.B. Hendrick, Toronto Developmental Anatomy Neuropathology

Managing Editor D.G. McLone, Chicago, 111. L.B. Rorke, Philadelphia, Pa.

D.H. Reigel, Pittsburgh, Pa. R.J. Lemire, Seattle, Wash. L. Becker, Toronto

Associate Editors J. Warkany, Cincinnati, Ohio F. Gilles, Los Angeles, Calif.

R.L. McLaurin, Cincinnati, Ohio Pediatric Neurology M. Norman, Vancouver

D.G. McLone, Chicago, 111. J.J. Volpe, St. Louis, Mo. Neuroimaging

T.P Naidich, Miami, Fla. M. Johnston, Ann Arbor, Mich. T.P. Naidich, Miami, Fla.

L.B. Rorke, Philadelphia, Pa. H. Singer, Baltimore, Md. B. Kendall, London

J.J. Volpe, St. Louis, Mo. D. Stumpf, Chicago, 111. C. Raybaud, Marseille

Pediatric Neuro-Oncology R.A. Zimmerman,

R.J. Packer, Philadelphia, Pa. Philadelphia, Pa.

C.C. Bailey, Leeds Pediatrics

L.E. Kun, Memphis, Tenn. D.B. Shurtleff, Seattle, Wash.

T. Tomita, Chicago, 111. Medical Media

Pediatric Neuro-Ophthalmology J. Vetter, Pittsburgh, Pa.

EC. Chu, St. Louis, Mo.

C.S. Hoyt, San Francisco, Calif.

Pediatric Neuropsychology

H.S. Levin, Galveston, Tex.

Pediatric Neurosurgery

R.L. McLaurin, Cincinnati, Ohio

D. Bruce, Dallas, Tex.

P. Chapman, Boston, Mass.

M. Edwards, San Francisco, Calif.

R. Humphreys, Toronto

J. Venes, Ann Arbor, Mich. 